\title{
Impaired STAT3-Dependent Upregulation of IL2R $\alpha$ in B Cells of a Patient With a STAT1 Gain-of-Function Mutation
}

\begin{abstract}
Menno C. van Zelm ${ }^{1,2,3 *}$, Julian J. Bosco ${ }^{2,3}$, Pei M. Aui ${ }^{1,3}$, Samuel De Jong ${ }^{1,3}$, Fiona Hore-Lacy ${ }^{2,3}$, Robyn E. O'Hehir ${ }^{2,3}$, Robert G. Stirling ${ }^{2,3}$ and Paul U. Cameron ${ }^{3,4,5 *}$

${ }^{1}$ Department of Immunology and Pathology, Central Clinical School, Monash University, Melbourne, VIC, Australia, ${ }^{2}$ Allergy, Asthma and Clinical Immunology Service, Department of Respiratory, Allergy and Clinical Immunology (Research), Central Clinical School, Monash University, The Alfred Hospital, Melbourne, VIC, Australia, ${ }^{3}$ The Jeffrey Modell Diagnostic and Research Centre for Primary Immunodeficiencies in Melbourne, Melbourne, VIC, Australia, ${ }^{4}$ Department of Infectious Diseases, Alfred Hospital and Monash University, Melbourne, VIC, Australia, ${ }^{5}$ The Peter Doherty Institute for Infection and Immunity, University of Melbourne and Royal Melbourne Hospital, Melbourne, VIC, Australia
\end{abstract}

\section{OPEN ACCESS}

Edited by:

Marta Rizzi

Freiburg University Medical Center, Germany

Reviewed by:

Satoshi Okada,

Hiroshima University, Japan Vassilios Lougaris,

University of Brescia, Italy

*Correspondence:

Menno C. van Zelm

menno.vanzelm@monash.edu

Paul U. Cameron

paul.cameron@unimelb.edu.au

Specialty section:

This article was submitted to

Primary Immunodeficiencies,

a section of the journal

Frontiers in Immunology

Received: 01 January 2019 Accepted: 22 March 2019

Published: 24 April 2019

Citation:

van Zelm MC, Bosco JJ, Aui PM, De Jong S, Hore-Lacy F, O'Hehir RE, Stirling RG and Cameron PU (2019) Impaired STAT3-Dependent Upregulation of IL2R $\alpha$ in B Cells of a

Patient With a STAT1 Gain-of-Function

Mutation. Front. Immunol. 10:768.

doi: 10.3389/fimmu.2019.00768
Heterozygous STAT1 gain-of-function (GOF) mutations form the most common genetic cause of chronic mucocutaneous candidiasis (CMC). In such patients, increased STAT1 function leads to impaired STAT3-dependent activation of IL-17A and IL-17F in T cells, thereby causing impaired Th17 responses to Candida. In spite of the critical role of STAT3 in IL-21 signaling in B cells, nearly all STAT1 GOF patients have normal or high serum IgG. We here present a 44 year-old male with childhood onset of CMC and antibody deficiency since early adulthood. Sequence analysis of STAT1 revealed a heterozygous missense mutation in the coiled-coil domain (p.D168E), which resulted in increased STAT1 phosphorylation of B-cells activated with IFN $\alpha$ and IFN $\gamma$. IL-21 induced STAT3 phosphorylation and nuclear localization were normal, but resulted in impaired upregulation of IL2R $\alpha$. This newly identified B-cell intrinsic impairment of STAT3 function could underlie the progressive development of hypogammaglobulinemia. Considering the high risk of bronchiectasis and irreversible organ damage, this case illustrates the need for monitoring of lgG levels and/or function in adult patients with STAT1 GOF mutations.

Keywords: chronic mucocutaneous candidiasis, hypogammaglobulinemia, STAT1, gain-of-function, STAT3, IL2R $\alpha$

\section{BACKGROUND}

Chronic mucocutaneous candidiasis (CMC) is a persistent or recurrent infection by Candida and typically affects the nails, skin, oral, and genital mucosae. In recent years, many cases have been shown to result from primary immunodeficiencies (PIDs) with impaired helper-T(h)17 cell immunity (1). This can be due to inhibitory autoantibodies against Th17 cytokines in patients with autosomal recessive (AR) polyendocrine syndrome type I (APS-1), or alternatively, inherited mutations that impair development and function of Th17 cells. Heterozygous STAT1 gain-offunction (GOF) mutations form the most common genetic cause of CMC with mutations found in more than $50 \%$ of patients (2-4). These mutations are typically found in exons 7-14 which encode the coiled-coil and DNA-binding domains. As a result, increased STAT1 phosphorylation occurs 
upon stimulation of immune cells with STAT1-activating cytokines, such as interferon (IFN) $\alpha$ and IFN $\gamma$. Importantly, increased STAT1 signaling reciprocally inhibits STAT3dependent cytokine production, which include IL-17A and IL-17F in T cells. Thus, STAT1 GOF predisposes to impaired Th17 responses to Candida $(2,4)$.

Patients with STAT1 GOF mutations often present with additional bacterial and viral complications. Furthermore, autoimmunity/autoinflammatory disease has been observed in $37 \%$ of patients in a large cohort study $(n=274)$, and several patients have been shown to develop solid tumors (3). Effects on B-cells and humoral immunity are variable. 19\% of 209 patients carried reduced total B cell numbers and $49 \%$ of the 53 patients examined had reduced memory B cell numbers. In addition, up to $23 \%$ of patients have impaired antibody responses to vaccinations with protein antigens, although only $3 \%$ have hypogammaglobulinemia $(3,5)$. As STAT3 is critical for IL21dependent signaling in T-cell dependent B-cell responses, it is possible that STAT1 GOF mutations affect antibody responses and humoral immunity by inadvertent repression of STAT3mediated transcription. We here identify a defect in STAT3dependent upregulation of IL2R $\alpha$ (CD25) in B cells of a patient with STAT1 GOF.

\section{METHODS}

\section{Ethics}

Diagnostic work-up of blood and laboratory research studies including genetics of the patient were carried out with approval of Human Research Ethics committee of The Alfred Hospital (Study 109/15) and obtained after written informed consent. In addition, the patient has consented to publication of the case report. Data from healthy controls were collected after written consent was obtained and with approval of the human ethics committee of Monash University (Study 2016-0289). All studies were performed in accordance with the Declaration of Helsinki.

\section{Flowcytometric Immunophenotyping and in vitro Cell Stimulation}

Patient and control subjects were included over a time period of 3 years. Standardized sample preparation, antibody staining, and flow cytometer instrument settings were used to ensure consistency in flow cytometry (6). In short, absolute counts of CD3+, CD4+ and CD8+ T cells, CD19+ B cells, and CD16+/CD56+ natural killer cells were obtained with a diagnostic lyse-no-wash protocol by using commercial Trucount tubes (BD Biosciences, San Jose, CA). For detailed 11-color flow cytometry, red blood cells were lysed with $\mathrm{NH}_{4} \mathrm{Cl}$ before incubation of 1-2 million nucleated cells for $15 \mathrm{~min}$ at room temperature in a total volume of $100 \mu \mathrm{L}$. After preparation, cells were measured on 4-laser flow cytometer (LSRII or LSRFortessa, BD Biosciences) by using standardized settings (6). Data were analyzed with FACSDiva (V8.0; BD Biosciences) and FlowJo software (v10) Naive and memory B-cells, and CD4+ T-cell subsets were defined as previously described (7).

Immortalization of patient's and control B cells with EBV derived from supernatant of the B95-8 cell line was performed as described previously (8). The EBV LCL were stimulated in vitro for $30 \mathrm{~min}$ with IFN $\alpha(10,000 \mathrm{U} / \mathrm{ml}$; pbl assay science), IFN- $\gamma$ (10,000 U/ml; Peprotech), or IL-21 $(50 \mathrm{ng} / \mathrm{ml}$; Lonza). Subsequently, the cells were stained with CD20-BV605 (clone 2H7; BioLegend) and Fixable Viability Stain 700 (BD Biosciences) prior to fixation, permeabilization, and staining with STAT1(pY701)-AF67 (clone 4a) and STAT3(pY705)-PE (clone 4/P-STAT3) according to manufacturer's instructions (BD Biosciences). Following acquisition on a 4-laser LSRII (BD Biosciences), live single cells that were positive for CD20 expression were analyzed for intracellular pSTAT1 and pSTAT3 expression (FlowJo v10). In addition, nuclear localization of pSTAT3 following $30 \mathrm{~min}$ IL-21 stimulation was determined in EBV LCL from the patient and from a healthy control using an imaging flow cytometer (Imagestream ${ }^{\mathrm{X}}$ MKII; Amnis/Millennium Science, Mulgrave, VIC, Australia) equipped with four lasers $(405,488,642$, and $785 \mathrm{~nm})$. Images $(60 \mathrm{x})$ were obtained from $>1,000$ cells per condition and similarity scores were derived for pSTAT3 and the nucleus (stained with Vybrant $^{\mathrm{TM}}$ DyeCycle $^{\mathrm{TM}}$ Violet; Thermo Scientific). Similarity scores for surface CD20 (BV605) and the nucleus were derived as negative control.

\section{Sequence Analysis of STAT1}

Following genomic DNA isolation from post-Ficoll granulocytes (GenElute Mammalian Genomic DNA Miniprep Kit, SigmaAldrich, St Louis, Mo), exons 7-14 of the STAT1 gene were PCR-amplified using previously published primers (9), and sequenced by the Micromon facility of Monash University on an Applied Biosystems 3730s DNA Analyzer (Thermo Fisher). Obtained sequences were aligned with the reference sequence from Ensembl using CLC Main Workbench 7 software.

\section{Molecular Analysis of Ig Gene Rearrangements}

RNA was isolated from post-Ficoll mononuclear cells of the patient with a GenElute mammalian RNA kit (SigmaAldrich) and reverse transcribed to cDNA with random primers (Invitrogen Life technologies). Rearranged IgG and IgA transcripts were amplified in a multiplex PCR approach using 4 different $I G H V$-family leader forward primers in combination with an IGHG-consensus or IGHA-consensus reverse primer $(10,11)$. PCR products were cloned into a pGEMT easy vector (Promega, Madison WI), amplified by colony PCR, and sequenced as above. Sequences were analyzed using the IMGT database (http://www.imgt.org/IMGT_vquest/vquest) to assign the $I G H V, I G H D$, and $I G H J$ genes and alleles, and to identify somatic hypermutations (SHM). Of each unique clone, the position and frequency of mutations were determined within the entire $I G H V$ gene (FR1-CDR1-FR2-CDR2-FR3). SHM were determined as variations on the best matched $\mathrm{V}$-gene and represented as the percentage of mutations of the total sequenced $\mathrm{V}$-gene nucleotides. The IgG and IgA receptor subclasses were determined using the $I G H$ reference sequence (NG_001019). All results of the patient were compared with previously generated data sets of controls (12). 


\section{CASE PRESENTATION}

\section{Clinical History}

We here present a 44 year-old male with a history of CMC treated since early childhood with azole antifungal agents. The patient is the second of three children from non-consanguineous parents. He has developed resistance to antifungal drugs including nystatin, fluconazole, and partially to voriconazole to which he had an allergic drug reaction of troublesome and persistent photodermatitis. He is currently controlled on posaconazole and amphotericin lozenges.

The CMC has been associated with the development of esophageal strictures requiring repeated dilation. At the age of 39 years this procedure was complicated by esophageal rupture and mediastinitis requiring a prolonged ICU admission. The esophageal rupture was treated surgically but subsequent investigations for recurrent stenosis led to diagnosis of esophageal cancer at age 40. He underwent esophageal resection a year later with clear surgical margins, followed by adjuvant chemotherapy which was truncated because of severe mucositis. Radiotherapy was commenced for this cancer due to poor prognosis in young age.

Shortly after diagnosis with esophageal cancer, the patient was started on G-CSF therapy (2 times $300 \mu \mathrm{g}$ per week) for almost 2 years (Dec 2014-July 2016). As the patient reported increased discomfort following discontinuation, G-CSF therapy was re-started a year later at age 43 years and is still current.

During early adulthood, the patient developed progressive hypogammaglobulinemia (Table 1) with poor vaccine responses and commenced IVIG replacement at age 35 . In spite of adequate trough IgG with monthly IVIG, he continues to suffer from recurrent lower respiratory tract infections requiring antibiotics and has been hospitalized on at least 4 occasions with bacterial infections, including salmonella gastroenteritis. He has required periodic courses of IV caspafungin for candida partially resistant to azoles.

\section{Identification of a Heterozygous Gain-of-Function Mutation in STAT1}

Given the severity of the CMC and the antibody deficiency, more detailed immunological work-up was performed in the context of a research study. Detailed flowcytometric immunophenotyping of the patient's B- and T-cells revealed a severe reduction in CD27+ memory B cells and low circulating numbers of Th17 cells at age 42 years following discontinuation of GCSF therapy (Table 1). As the patient did not have typical clinical associations of APS-1, a STAT1 GOF mutation was considered and genetic analysis of STAT1 exons 7-14 was performed on DNA of the patient. Sanger sequencing revealed a heterozygous variant in exon $7(\mathrm{c} .504 \mathrm{~T}>\mathrm{A})$ resulting in a missense mutation in the coiled-coil domain (p.D168E) (Figure 1A). The same mutation has been previously described in a 5 year old female patient, but was not functionally addressed (3). To examine the effects of the mutation, we studied phosphorylation of STAT1 in EBV-immortalized B-lymphocytes of the patient. Thirty minutes after stimulation with either IFN $\alpha$ or IFN $\gamma$, the patient's cells showed increased levels of pSTAT1
TABLE 1 | Immunological data.

\begin{tabular}{|c|c|c|c|}
\hline \multirow[t]{2}{*}{ Laboratory measurement } & \multicolumn{2}{|c|}{ Patient } & \multirow[t]{2}{*}{ Normal range* } \\
\hline & 35 year & 42 year & \\
\hline \multicolumn{4}{|l|}{ SERUM IG LEVELS (g/L) } \\
\hline $\lg G$ & 3.4 & - & $7.0-15.5$ \\
\hline $\lg G 1$ & 1.8 & - & $3.8-9.3$ \\
\hline $\lg G 2$ & 1.8 & - & $2.4-7.0$ \\
\hline $\lg 33$ & 0.3 & - & $0.22-1.76$ \\
\hline $\lg G 4$ & 0.1 & - & $0.04-0.86$ \\
\hline $\lg A$ & 1.6 & - & $0.76-3.9$ \\
\hline $\lg \mathrm{M}$ & 0.3 & - & $0.45-2.3$ \\
\hline \multicolumn{4}{|c|}{ LYMPHOCYTE SUBSETS (CELLS/ $\mu$ I BLOOD) } \\
\hline B cells & 51 & 103 & $76-608$ \\
\hline Transitional & - & 0.9 & $0.4-29$ \\
\hline Naive mature & - & 87 & $31-398$ \\
\hline $\operatorname{lgD}+$ memory & - & 7.1 & $3.4-79$ \\
\hline IgD- memory & - & 2.4 & $12-114$ \\
\hline T cells & 1,248 & 1,013 & $773-2,757$ \\
\hline CD8 & 576 & 475 & 243-950 \\
\hline CD4 & 624 & 409 & $307-1,600$ \\
\hline Tfh (CD45RA-CXCR5+) & - & 26 & $16-175$ \\
\hline Th17 (CD45RA-CCR6-CCR4+CXCR3-) & - & 7.4 & $11-98$ \\
\hline
\end{tabular}

"For cell subsets: 5-95\% of adult controls; $B$ cells, $n=44 ; T$ cells, $n=34$. Values below normal range are depicted in bold font.

confirming a GOF phenotype as a result of the D168E missense mutation (Figure 1B).

\section{T-Helper and T-Follicular Helper Cell Subsets}

Given that the patient reported beneficial effects of G-CSF treatment, we retrospectively analyzed immune cells prior-to and during the treatment period. Extensive follow-up of total leukocyte and neutrophil count showed a general increase in numbers during therapy (Figure 2A). Three stored PBMC samples were available for detailed T-cell immunophenotyping, and reporting of relative frequencies of Th17 and Th cells. Th17 cell frequencies were within the normal range on only 1 occasion under G-CSF therapy, whereas Tfh cell frequencies were not below the normal range (Figure 2B). Hence, G-CSF therapy was associated with normalization of Th17 cells on at least one occasion.

\section{STAT3 Signaling Defect in B Cells}

To gain more insight into the nature of the hypogammaglobulinemia and reduced memory $B$ cells in the patient, we first quantified SHM in IgG transcripts from blood B cells. Overall, SHM levels were normal. However, IgG3 transcripts of the patient contained negligible SHM, in contrast to IgG1 and IgG2 (Figure 3A). Further analysis of the IgG transcripts demonstrated a predominant usage of IgG3 compared to IgG2 (Figure 3B).

T-cell dependent B-cell responses critically depend on IL21R signaling via STAT3. As STAT1 GOF mutations can 

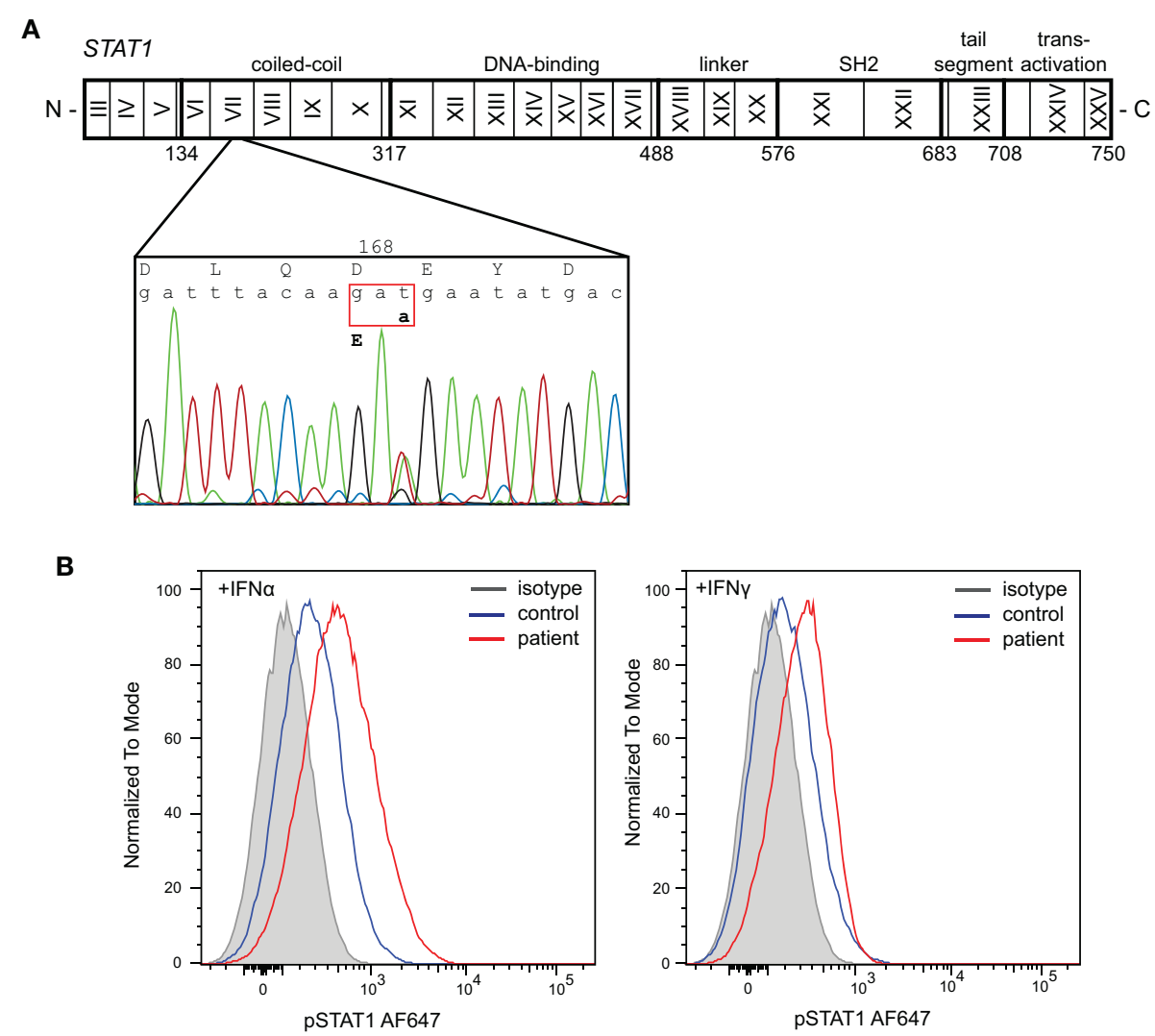

FIGURE 1 | Identification of a heterozygous mutation in STAT1 leading to a gain of function. (A) Sanger sequencing revealed a heterozygous c.504T>A mutation in exon 7 resulting in a missense mutation in the coiled-coil domain (p.D168E). (B) Increased phosphorylation of STAT1 following in vitro stimulation of patient's EBV-LCL with IFN $\alpha$ and IFN $\gamma$.

inhibit STAT3 activity, we here questioned whether the patient's B cells had intrinsically impaired STAT3 responsiveness. Indeed, in EBV-immortalized B cells from the patient, IL21 stimulation normally induced STAT3 phosphorylation (Figure 3C). Moreover, nuclear localization studies with imaging flowcytometry revealed normal nuclear localization of pSTAT3 after IL-21 stimulation as well (Figure 3D). Therefore, we next evaluated functional STAT3 signaling by evaluation of expression of CD25, the IL2R $\alpha$ chain, which is a direct target of STAT3 in B cells (13). Following $24 \mathrm{~h}$ incubation with IL-21, EBV-LCL from a healthy control upregulated CD25 surface expression (Figure 3E). In contrast, EBV-LCL from the patient had lower levels of CD25 expression. These findings are consistent with previous finding that STAT3 activity was inhibited by STAT1 GOF at the target gene activation level, but not upstream of that (14).

\section{DISCUSSION}

We here report a patient with STAT1 GOF and adult-onset antibody deficiency in the context of reduced total and memory B cells and impaired SHM and class switching to IgG2. Despite the known inhibition of STAT3 function due to STAT1 GOF, this has not been extensively addressed in previous studies. In contrast to the highly penetrant defects in Th17 function, the impact of STAT1 GOF mutations on B-cell function and antibody responses is variable among reported patients (3). In B cells from our patient, we confirmed that following stimulation with IL21, STAT3 phosphorylation and nuclear localization were not affected, but that activation of expression of the target gene encoding CD25 (IL-2R $\alpha$ ) was impaired. Hence, these B-cells will not be optimally sensitized to the stimulatory effects of IL-2 (13), and subject to suboptimal humoral immune responses.

Using in vitro functional analysis, we showed that the heterozygous STAT1 D168E mutation in our patient had a dominant GOF effect on STAT1 phosphorylation. The increased amount of pSTAT1 protein following activation is generally assumed to be the result of a larger fraction of the mutant STAT1 being phosphorylated. However, as we were unable to measure total STAT1 protein, it remains possible that the mutant STAT1 is expressed at a higher level than the wild type, providing more total protein to be phosphorylated upon activation.

The same D168E mutation has been identified previously in a 5 year-old from Moroccan descent (patient 167 from kindred 111), but was not functionally assessed (3). The Moroccan girl did not present with hypogammaglobulinemia (IgG, $15.86 \mathrm{~g} / \mathrm{L}$ ), 


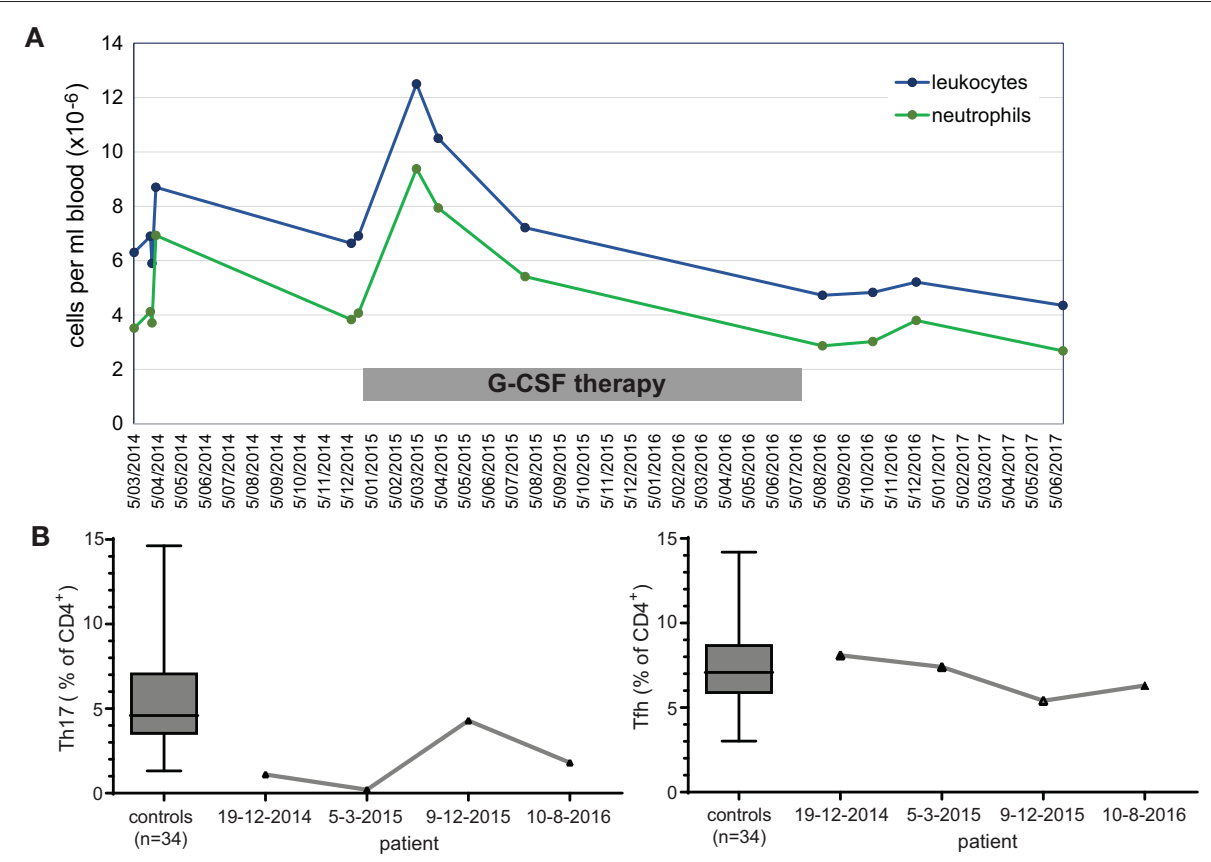

FIGURE 2 | Immunological effects of immunotherapy with G-CSF. (A) Longitudinal measurements of blood leukocyte and neutrophil cell counts over a 3 year interval. (B) Frequencies of Th17 and Tfh cell subsets within total CD4T cells prior to, during (2 timepoints; 2015) and after discontinuation of G-CSF therapy.

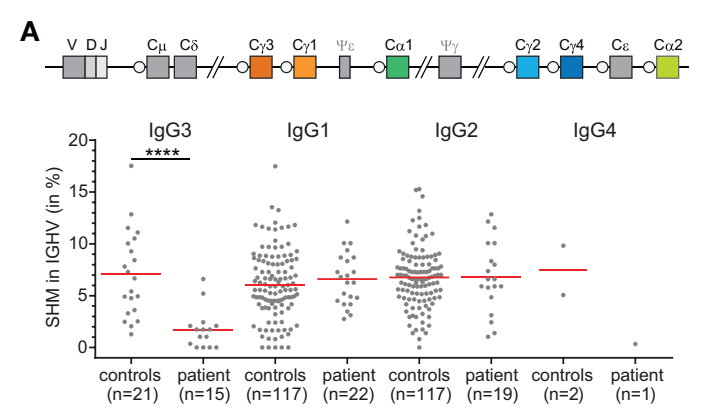

D

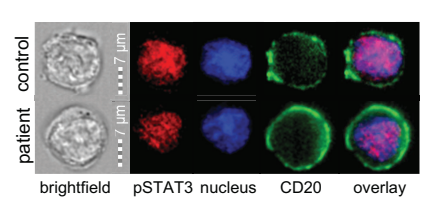

B

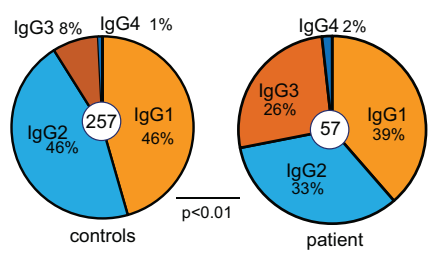

E

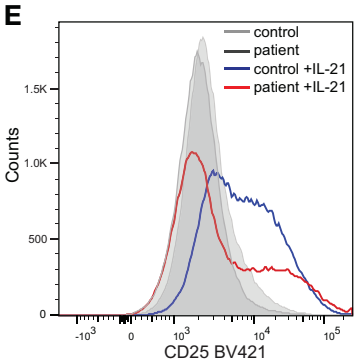

C

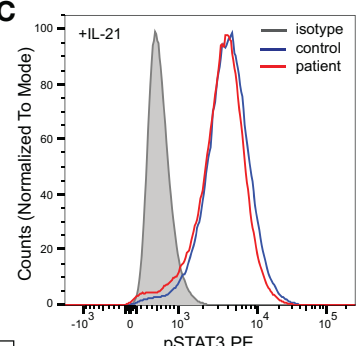

PSTAT3 PE

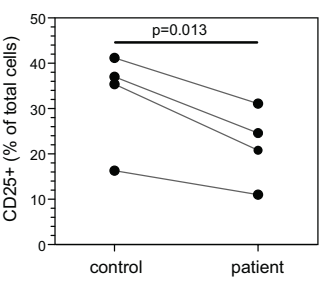

FIGURE 3 | B-cell defects in STAT1 GOF. (A) Somatic hypermutation levels in IgG subclass transcripts of the patient and controls. (B) Relative distributions of IgG subclasses of unique transcripts. (C) STAT3 phosphorylation in EBV-LCL upon stimulation with IL-21. (D) Nuclear localization of pSTAT3 in EBV-LCL following stimulation with IL-21 as determined with the AMNIS ImageStream. Example images are shown on the left, and the collated events as overlays for nuclear localization scores on the right. (E) CD25 (IL2R $\alpha$ ) expression on EBV-LCL following stimulation with IL-21. Overlays are shown for one experiment to illustrate on the left and the combined data from 4 independent experiments on the right. Statistics: Paired $t$-test.

nor did our patient before early adulthood. It is therefore possible that patients with STAT1 GOF mutations are susceptible for a progressive decline in serum IgG levels and consequent antibody deficiency. This decline in antibody responses could be the accumulated effect of Tfh cell defects and the B-cell intrinsic defect to respond to IL-21. As shown by our in vitro data, this potentially mimics STAT3 LOF mutations with impaired activation of STAT3 target genes (13). 
In this patient the STAT1 GOF may have directly contributed to the development of esophageal cancer. Early onset cancers were present in $6 \%$ of patients in the large series (3), and suggest that immunological abnormalities including Th17 and B cell function may also be associated with risk for malignancies.

Prior to the genetic diagnosis of STAT1 GOF, the patient was started on experimental G-CSF treatment based on the well-described anti candida function of this cytokine, as well as indications in the literature of a beneficial effect of adjunctive immunotherapy for the treatment of disseminated candidiasis (15). G-CSF therapy in our patient did increase Th17 cell frequencies, but did not resolve the candidiasis. The latter is in line with a recent report of an impaired capacity for killing of C. albicans by G-CSF recruited neutrophils (16). Moreover, it was previously reported that Th17 responses in STAT1 GOF patients were not restored by G-CSF immunotherapy (17). We here show a partial effect of G-CSF resulting in a clinical benefit. Increasing evidence is accumulating that Jak inhibitors are successful in resolving candidiasis in STAT1 GOF patients (18-20), but this is not entirely risk-free (21). Unfortunately, we have not yet been able to obtain compassionate access to Jak inhibitors for our patient, and as he does not experience adverse effects of G-CSF therapy this therapy is still continued.

\section{CONCLUDING REMARKS}

We describe a B-cell intrinsic impairment of STAT3 function in a patient with a STAT1 GOF mutation and progressive development of hypogammaglobulinemia. Currently, a minority of the reported patients with STAT1 GOF mutations suffer from hypogammaglobulinemia with a larger proportion showing impaired responses to vaccination (3). Considering the high risk of bronchiectasis and irreversible organ damage $(22,23)$, this case

\section{REFERENCES}

1. Puel A, Cypowyj S, Marodi L, Abel L, Picard C, Casanova JL. Inborn errors of human IL-17 immunity underlie chronic mucocutaneous candidiasis. Curr Opin Allergy Clin Immunol. (2012) 12:616-22. doi: 10.1097/ACI.0b013e328358cc0b

2. Liu L, Okada S, Kong XF, Kreins AY, Cypowyj S, Abhyankar A, et al. Gain-of-function human STAT1 mutations impair IL-17 immunity and underlie chronic mucocutaneous candidiasis. J Exp Med. (2011) 208:1635-48. doi: $10.1084 /$ jem. 20110958

3. Toubiana J, Okada S, Hiller J, Oleastro M, Lagos Gomez M, Aldave Becerra JC, et al. Heterozygous STAT1 gain-of-function mutations underlie an unexpectedly broad clinical phenotype. Blood. (2016) 127:3154-64. doi: 10.1182/blood-2015-11-679902

4. van de Veerdonk FL, Plantinga TS, Hoischen A, Smeekens SP, Joosten LA, Gilissen C, et al. STAT1 mutations in autosomal dominant chronic mucocutaneous candidiasis. N Engl J Med. (2011) 365:54-61. doi: 10.1056/NEJMoa1100102

5. Kobbe R, Kolster M, Fuchs S, Schulze-Sturm U, Jenderny J, Kochhan $\mathrm{L}$, et al. Common variable immunodeficiency, impaired neurological development and reduced numbers of $\mathrm{T}$ regulatory cells in a 10-yearold boy with a STAT1 gain-of-function mutation. Gene. (2016) 586:234-8. doi: 10.1016/j.gene.2016.04.006 illustrates the need for monitoring of IgG levels and/or function in adult patients with STAT1 GOF mutations.

\section{ETHICS STATEMENT}

Diagnostic work-up of blood and laboratory research studies including genetics of the patient were carried out with approval of Human Research Ethics committee of The Alfred Hospital (Study 109/15) and obtained after written informed consent. Data from healthy controls were collected after written consent was obtained and with approval of the human ethics committee of Monash University (Study 2016-0289). All studies were performed in accordance with the Declaration of Helsinki.

\section{AUTHOR CONTRIBUTIONS}

MvZ, JB, and PC designed and wrote the manuscript. PA and SDJ performed experiments. FH-L, RO, and RS contributed to essential discussion of the paper. All authors critically read and commented on manuscript drafts and approved of the final version.

\section{FUNDING}

This work was supported by The Jeffrey Modell Foundation, and by a National Health and Medical Research Council Senior Research Fellowship (GNT1117687) to MvZ.

\section{ACKNOWLEDGMENTS}

We gratefully acknowledge technical support from Dr. Geza Paukovic and Steven Lim (AMREP Flow Cytometry Core Facility), and the support of Dr. Raffi Gugasyan and Mr. Jasper (Burnet Institute) with imaging flowcytometry. We thank Dr. Anne Puel (Paris, France) for sharing unpublished data.

6. Kalina T, Flores-Montero J, van der Velden VH, Martin-Ayuso M, Bottcher S, Ritgen M, et al. EuroFlow standardization of flow cytometer instrument settings and immunophenotyping protocols. Leukemia. (2012) 26:1986-2010. doi: 10.1038/leu.2012.122

7. Heeringa JJ, Karim AF, van Laar JAM, Verdijk RM, Paridaens D, van Hagen PM, et al. Expansion of blood IgG4(+) B, TH2, and regulatory $\mathrm{T}$ cells in patients with IgG4-related disease. J Allergy Clin Immunol. (2018) 141:1831-1843.e1810. doi: 10.1016/j.jaci.2017. 07.024

8. van Zelm MC, Smet J, Adams B, Mascart F, Schandene L, Janssen F, et al. CD81 gene defect in humans disrupts CD19 complex formation and leads to antibody deficiency. J Clin Invest. (2010) 120:1265-74. doi: 10.1172/JCI39748

9. Chapgier A, Kong XF, Boisson-Dupuis S, Jouanguy E, Averbuch D, Feinberg J, et al. A partial form of recessive STAT1 deficiency in humans. J Clin Invest. (2009) 119:1502-14. doi: 10.1172/JCI37083

10. Berkowska MA, Schickel JN, Grosserichter-Wagener C, de Ridder D, Ng YS, van Dongen JJ, et al. Circulating human CD27-IgA + memory B cells recognize bacteria with polyreactive Igs. J Immunol. (2015) 195:1417-26. doi: 10.4049/jimmunol.1402708

11. Tiller T, Meffre E, Yurasov S, Tsuiji M, Nussenzweig MC, Wardemann H. Efficient generation of monoclonal antibodies from single human B cells by single cell RT-PCR and expression vector cloning. J Immunol Methods. (2008) 329:112-24. doi: 10.1016/j.jim.2007.09.017 
12. de Jong BG, IJspeert $H$, Marques L, van der Burg $M$, van Dongen JJ, Loos BG, et al. Human IgG2- and IgG4-expressing memory B cells display enhanced molecular and phenotypic signs of maturity and accumulate with age. Immunol Cell Biol. (2017) 95:744-52. doi: 10.1038/icb.2017.43

13. Berglund LJ, Avery DT, Ma CS, Moens L, Deenick EK, Bustamante J, et al. IL-21 signalling via STAT3 primes human naive B cells to respond to IL-2 to enhance their differentiation into plasmablasts. Blood. (2013) 122:3940-50. doi: 10.1182/blood-2013-06-506865

14. Zheng J, van de Veerdonk FL, Crossland KL, Smeekens SP, Chan CM, Al Shehri T, et al. Gain-of-function STAT1 mutations impair STAT3 activity in patients with chronic mucocutaneous candidiasis (CMC). Eur J Immunol. (2015) 45:2834-46. doi: 10.1002/eji.201445344

15. van de Veerdonk FL, Kullberg BJ, Netea MG. Adjunctive immunotherapy with recombinant cytokines for the treatment of disseminated candidiasis. Clin Microbiol Infect. (2012) 18:112-9. doi: 10.1111/j.1469-0691.2011.03676.x

16. Gazendam RP, van de Geer A, van Hamme JL, Tool AT, van Rees DJ, Aarts $\mathrm{CE}$, et al. Impaired killing of Candida albicans by granulocytes mobilized for transfusion purposes: a role for granule components. Haematologica. (2016) 101:587-96. doi: 10.3324/haematol.2015.136630

17. van de Veerdonk FL, Koenen HJ, van der Velden WJ, van der Meer JW, Netea MG. Immunotherapy with G-CSF in patients with chronic mucocutaneous candidiasis. Immunol Lett. (2015) 167:54-6. doi: 10.1016/j.imlet.2015.05.008

18. Forbes LR, Vogel TP, Cooper MA, Castro-Wagner J, Schussler E, Weinacht $\mathrm{KG}$, et al. Jakinibs for the treatment of immune dysregulation in patients with gain-of-function signal transducer and activator of transcription 1 (STAT1) or STAT3 mutations. J Allergy Clin Immunol. (2018) 142:1665-9. doi: 10.1016/j.jaci.2018.07.020

19. Higgins E, Al Shehri T, McAleer MA, Conlon N, Feighery C, Lilic D, et al. Use of ruxolitinib to successfully treat chronic mucocutaneous candidiasis caused by gain-of-function signal transducer and activator of transcription 1 (STAT1) mutation. J Allergy Clin Immunol. (2015) 135:551-3. doi: 10.1016/j.jaci.2014.12.1867

20. Meesilpavikkai K, Dik WA, Schrijver B, Nagtzaam NMA, Posthumusvan Sluijs SJ, van Hagen PM, et al. Baricitinib treatment in a patient with a gain-of-function mutation in signal transducer and activator of transcription 1 (STAT1). J Allergy Clin Immunol. (2018) 142:328-330.e322. doi: 10.1016/j.jaci.2018.02.045

21. Zimmerman O, Rosler B, Zerbe CS, Rosen LB, Hsu AP, Uzel G, et al. Risks of ruxolitinib in STAT1 gain-of-function-associated severe fungal disease. Open Forum Infect Dis. (2017) 4:ofx202. doi: 10.1093/ofid/ofx202

22. Breuer O, Daum H, Cohen-Cymberknoh M, Unger S, Shoseyov D, Stepensky P, et al. Autosomal dominant gain of function STAT1 mutation and severe bronchiectasis. Respir Med. (2017) 126:39-45. doi: 10.1016/j.rmed.2017.03.018

23. Huh HJ, Jhun BW, Choi SR, Kim YJ, Yun SA, Nham E, et al. Bronchiectasis and recurrent respiratory infections with a de novo STAT1 gain-offunction variant: first case in Korea. Yonsei Med J. (2018) 59:1004-7. doi: 10.3349/ymj.2018.59.8.1004

Conflict of Interest Statement: The authors declare that the research was conducted in the absence of any commercial or financial relationships that could be construed as a potential conflict of interest.

Copyright (c) 2019 van Zelm, Bosco, Aui, De Jong, Hore-Lacy, O'Hehir, Stirling and Cameron. This is an open-access article distributed under the terms of the Creative Commons Attribution License (CC BY). The use, distribution or reproduction in other forums is permitted, provided the original author(s) and the copyright owner(s) are credited and that the original publication in this journal is cited, in accordance with accepted academic practice. No use, distribution or reproduction is permitted which does not comply with these terms. 